\title{
e-Migrinter
}

17 | 2018

Enseigner les migrations internationales

\section{Les migrations dans la presse quotidienne en classe de Quatrième}

\section{Christel Maridet-Tjon-A-Tai et Antoine Dumont}

\section{(2) OpenEdition}

Journals

Édition électronique

URL : https://journals.openedition.org/e-migrinter/1109

DOI : 10.4000/e-migrinter. 1109

ISSN : 1961-9685

Éditeur

UMR 7301 - Migrinter

Référence électronique

Christel Maridet-Tjon-A-Tai et Antoine Dumont, « Les migrations dans la presse quotidienne en classe de Quatrième », e-Migrinter [En ligne], 17 | 2018, mis en ligne le, consulté le 20 mai 2021. URL : http:// journals.openedition.org/e-migrinter/1109; DOI : https://doi.org/10.4000/e-migrinter.1109

Ce document a été généré automatiquement le 20 mai 2021.

Tous droits réservés 


\title{
Les migrations dans la presse quotidienne en classe de Quatrième
}

\author{
Christel Maridet-Tjon-A-Tai et Antoine Dumont
}

1 Le contenu enseigné dans cette "Semaine de la presse » est celui du programme de géographie du cycle 4 et de l'éducation morale et civique, adossé à des faits d'actualité récente. Les compétences visées sont notamment liées à la pédagogie de l'éducation aux médias et à l'information, à la pédagogie de groupe et à l'expression orale.

2 L'intérêt de cette expérience est d'être pluridisciplinaire. Il s'agissait autant de développer un regard géographique sur les migrations que de saisir les migrations comme prétexte à un travail sur la presse, à l'apprentissage de sa lecture, à l'expression des sensibilités des élèves et l'approfondissement de leurs capacités de jugement, de sélection des informations, voire d'esprit critique. Mais comment enseigner les migrations à travers la presse? Pour y répondre, nous présenterons le contexte, les objectifs, les moyens et le déroulement de cette séquence, avant d'aborder les obstacles rencontrés et les améliorations possibles.

\section{Le contexte : un nouveau collège public en zone périurbaine}

3 Le collège Frida Kahlo a ouvert ses portes à la rentrée 2016. Il s'agit d'un établissement public accueillant 220 élèves environ et comptant une petite dizaine de professeurs titulaires de leur poste. L'équipe pédagogique est donc réduite et récente, ses membres apprenant à travailler ensemble tout au long de l'année. Ce travail en équipe est d'ailleurs pour partie devenu obligatoire à cette rentrée 2016, la réforme du collège imposant la mise en place d'enseignements pratiques interdisciplinaires (EPI). En classe de 4e, deux EPI ont été réalisés, sur la justice au premier semestre et sur la ville au second semestre. Ces EPI ont impliqué, en amont, une longue concertation entre professeurs de différentes disciplines. L'enseignement des migrations n'ayant pas été intégré à ces EPI, nous avons décidé d'en faire l'objet de la semaine de la presse en 4e. 
4 «La semaine de la presse et des Médias dans l'école » existe depuis 1989. En 2017, c'était sa $28 \mathrm{e}$ édition. C'est une semaine nationale qui « permet aux élèves depuis plus de vingt ans de mieux connaître l'univers des médias et de comprendre ses enjeux culturels et démocratiques. Les écoles, collèges et lycées inscrits participent du 20 au 25 mars 2017 à cette semaine organisée par le Centre de liaison de l'enseignement et des médias d'information (Clemi). $»^{1}$

5 Ce sont souvent les professeurs documentalistes qui effectuent cette inscription, qui permet notamment à l'établissement de bénéficier d'un échantillon conséquent d'exemplaires de presse d'horizons et de genres différents (journaux, magazines...). Mais le professeur documentaliste se doit aussi d'impulser une dynamique interdisciplinaire autour de cette semaine thématique. D'ailleurs, l'Éducation aux Médias et à l'Information fait partie de ses missions prioritaires, ainsi qu'il est précisé dans la nouvelle circulaire régissant leurs missions ${ }^{2}$.

6 Le collège est situé à Pontchâteau, une commune de 10000 habitants située dans une zone périurbaine, au Nord de la Brière et dont la plupart des actifs travaillent dans le bassin d'emploi de Saint-Nazaire, située à $30 \mathrm{~km}$, plutôt que dans les agglomérations de Vannes et Nantes, situées à $50 \mathrm{~km}$. Les élèves habitant Pontchâteau sont peu nombreux. La majorité habite les communes rurales alentour. Il y a très peu de mixité sociale et ethnique au sein de l'établissement. Les effectifs des classes de $4 \mathrm{e}$ varient de 25 à 27 élèves.

\section{Les objectifs}

7 L'expérience présentée ici avait pour objectif de croiser trois types d'enseignements au collège : l'éducation aux médias et à l'information, l'éducation morale et civique et, bien sûr, la géographie. Le but était de faire travailler les élèves sur certaines des compétences du Socle commun de connaissances, de compétences et de culture redéfinies par la dernière réforme du collège ${ }^{3}$.

\section{L'éducation aux médias et à l'information (EMI)}

8 En faisant travailler les élèves sur des articles de la presse quotidienne régionale, il s'agissait d'abord de les initier, pour la plupart, à la lecture d'un journal, à sa structure et son format spécifiques. L'objectif était donc qu'ils apprennent à identifier un article de presse, à le reconnaître à partir de certains indices et, secondairement, à en dégager les principales informations factuelles.

9 Ce faisant, l'activité contribuait à l'EMI, présentée dans les textes officiels comme une spécificité du cycle 4 (5e, $4 \mathrm{e}, 3 \mathrm{e})$ :

«Dans une société marquée parl'abondance des informations, les élèves apprennent à devenir des usagers des médias et d'Internet conscients de leurs droits et devoirs et maîtrisant leur identité numérique, à identifier et évaluer, en faisant preuve d'esprit critique, les sources d'information à travers la connaissance plus approfondie d'un univers médiatique et documentaire en constante évolution. Ils utilisent des outils qui leur permettent d'être efficaces dans leurs recherches » (les passages soulignés sont ceux les plus concernés par l'activité décrite ici). 
10 L'EMI est mentionnée dans les cinq domaines de compétences du nouveau socle, en particulier dans le domaine 2 , où on souligne sa contribution à l'acquisition de méthodes et outils pour apprendre. En effet, l'EMI "pousse à s'interroger sur la fiabilité, la pertinence d'une information, à distinguer les sources selon leur support. » Dans le domaine 1, l'EMI participe à la maîtrise de la langue française et des «systèmes d'information et de communication à travers lesquels se construisent le rapport aux autres et l'autonomie. "

\section{Léducation morale et civique (EMC)}

Ce rapport à autrui est au centre de l'EMC, qui s'est substituée à l'éducation (collège) et l'instruction (école) civiques en 2015'. L'un des objectifs de l'EMC en cycle 4 est d'«identifier et exprimer en les régulant ses émotions et ses sentiments » en réfléchissant, par exemple, «aux différentes formes de racismes et de discriminations. »Cet objectif est repris en termes de compétences à acquérir dans le domaine $3 \mathrm{du}$ socle, intitulé « la formation de la personne et du citoyen ", qui invite à « développe[r] le vocabulaire des émotions et du jugement, la sensibilité et la pensée, concernant notamment les questions socialement vives et l'actualité ». Il s'agissait donc ici de confronter des élèves de $4 \mathrm{e}$ aux difficultés et aux drames souvent vécus par les migrants, afin de les amener à interroger les notions de justice, de sécurité, d'hospitalité et de solidarité.

L'autre objectif civique de cette expérience est lié à la capacité de jugement et d'esprit critique, déjà au cœur de l'EMI. Les programmes d'EMC indiquent que c'est précisément « dans le cadre de la semaine de la presse » que peut être «mener une réflexion sur la place et la diversité des médias dans la vie sociale et politique ». Cette interdépendance entre EMI et EMC est confirmée dans le domaine 3 du socle, précisant que :

«l'éducation aux médias et à l'information oblige à questionner les enjeux

démocratiques liés à l'information journalistique et aux réseaux sociaux. »

\section{La géographie}

Les compétences à travailler en géographie sont nombreuses et appartiennent souvent à différents domaines du socle. Celles qui étaient visées ici n'étaient pas disciplinaires au sens strict (se repérer dans l'espace, réaliser des productions cartographiques, etc.) mais liées à l'identification, l'analyse et la compréhension d'un document écrit, ainsi qu'à la coopération au sein d'un groupe d'élèves.

14 Les connaissances visées sont précisées (et soulignées) dans le tableau ci-dessous tiré $\mathrm{du}$ BOEN $^{5}$.

\begin{tabular}{|l|l|}
\hline $\begin{array}{l}\text { Repères annuels } \\
\text { de programmation }\end{array}$ & Démarches et contenus d'enseignement \\
\hline $\begin{array}{l}\text { Thème 2 } \\
\text { Les mobilités } \\
\text { humaines } \\
\text { transnationales }\end{array}$ & $\begin{array}{l}\text { Il est essentiel de montrer aux élèves l'importance des grands mouvements } \\
\text { transnationaux de population que le monde connaît et qui sont d'une ampleur } \\
\text { considérable. }\end{array}$ \\
\hline
\end{tabular}




\begin{tabular}{|c|c|}
\hline $\begin{array}{l}\text { Un monde de } \\
\text { migrants. }\end{array}$ & $\begin{array}{l}\text { Les migrations transnationales, dont les motivations peuvent être } \\
\text { extrêmement variées (Erasmus, suite de conflits, crise climatique, raisons } \\
\text { économiques...), sont souvent au centre de l'actualité et il est important que } \\
\text { les élèves comprennent que cette géographie des migrations n'est pas centrée } \\
\text { sur la seule Europe, ni marquée par les seuls mouvements des «Suds » vers les } \\
\text { « Nords », mais comporte aussi des foyers de migrations intracontinentales } \\
\text { sud-sud. }\end{array}$ \\
\hline $\begin{array}{l}\text { Le tourisme et ses } \\
\text { espaces. }\end{array}$ & $\begin{array}{l}\text { Quant au tourisme international, il constitue désormais le mouvement de } \\
\text { population le plus massif que le monde ait jamais connu; il est porteur d'effets } \\
\text { économiques, sociaux et territoriaux très importants. } \\
\text { Chaque sous-thème est abordé par une étude de cas locale ou régionale, au } \\
\text { choix du professeur, mise en perspective à l'échelle mondiale, afin de pouvoir } \\
\text { monter en généralité. } \\
\text { Ce thème permet des liens avec le programme d'histoire de } 4 \mathrm{e} \text {. }\end{array}$ \\
\hline
\end{tabular}

\section{Les moyens}

\section{La programmation}

L'enseignement présenté ici correspond au thème 2 du programme, dont il forme l'introduction. Il est placé en milieu d'année : auparavant, les élèves auront découvert "l'urbanisation du monde" (thème 1 , en janvier/février) et, en histoire, "les conquêtes et sociétés coloniales » (février/mars), deux questions intimement liées aux dynamiques migratoires.

Le programme d'histoire-géographie du cycle $4^{6}$ ne fixe plus de volume horaire par thème :

« Les enseignants déterminent le volume horaire qu'ils consacrent à chaque thème ou sous-thème en fonction des démarches pédagogiques qu'ils souhaitent mettre en œuvre ».

Mais il insiste sur leur mise en œuvre «de manière équilibrée, à parts égales ». Un calcul sur 30 semaines effectives de cours, comptant chacune 3 heures d'histoiregéographie-EMC, donne donc 40 heures d'histoire, 40 heures de géographie et 16 heures d'éducation morale et civique. Le thème 2 consacré en grande partie à « un monde de migrants " peut donc être traité en 13 à 14 heures.

L'emploi du temps au collège engendre une série de contraintes sur la programmation. Nous tenions à ce que la séquence sur les migrations débute pendant la semaine de la presse, du 20 au 25 mars, par un travail de groupe au CDI. Mais il a fallu tenir compte des deux semaines de vacances de printemps (du 8 au 24 avril), des jours fériés et des cours annulés pour de multiples raisons (liées aux EPI, à la vie scolaire, etc.). Enfin, la séquence devait s'achever et être évaluée avant le $14 \mathrm{mai}$, date du départ des élèves de $4 \mathrm{e}$ pour un voyage scolaire à Londres. Au total, les élèves de $4 \mathrm{e}$ ont bénéficié de 13 (4e B) à 15 heures $(4 \mathrm{e} C)$ d'enseignement des migrations du 20 mars au 12 mai. 
Figure $\mathrm{n}^{\circ} 1$ : Calendrier de la séquence sur les migrations

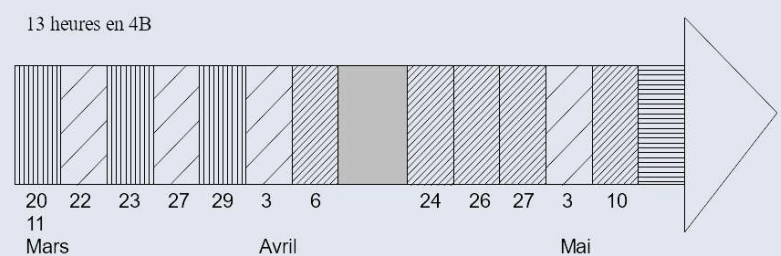

15 heures en $4 \mathrm{C}$

Mai

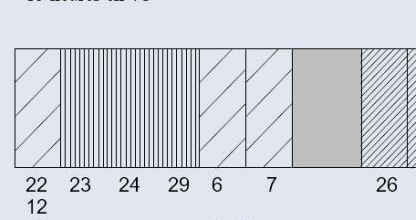

Mars

Avril

$\begin{array}{llllllll}26 & 27 & 28 & 3 & 4 & 5 & 10 & 11\end{array}$

Mai

Légende

||||| CDI : Travail sur la structure d'un article de presse (en classe entière), puis exposé oral (en groupe) sur un article de Onest-France sur les migrants.

Classe et salle multimedia : Travail sur les sources de l'infomation (1h); écriture (1h 4B) puis saisie d'un article de presse en histoire sur l'Algérie (1h): fin des exposés oraux ( $\mathrm{lh} 4 \mathrm{C})$; débat sur l'ouverture des frontières aux migrations ( $1 \mathrm{~h})$.

Classe : cours de géographie sur les mobilités humaines transnationales.

聖 Évaluation sommative de géographie.

Vacances scolaires.

Source : auteurs.

\section{Le corpus}

Le corpus étudié contenait 19 articles de presse tous parus dans le journal quotidien Ouest-France du 5 janvier au 20 mars 2017 dans les rubriques Monde/Europe (10 articles), Monde/France (cinq), Pays-de-la-Loire (un), Nantes métropole (un), Pays de Retz (deux). Un corpus plus important au départ (36 articles) avait été pré-sélectionné par la collègue documentaliste puis soumis au professeur d'histoire-géographie. Mais les élèves ont ensuite effectué un choix parmi ces articles qui en a réduit le nombre par deux. Le document ci-dessous récapitule ces articles. Il n'a pas été distribué aux élèves puisqu'il donnait certaines réponses attendues, mais il nous servait de guide.

\section{Les articles sélectionnés par les élèves}

\begin{tabular}{|l|l|l|l|l|l|}
\hline Élèves/classes & Titre & Date & Rubrique & Page & Angle \\
\hline H. garçon $4 \mathrm{C}$ & $\begin{array}{l}\text { Terminus pour } \\
\text { les néonazis } \\
\text { slovaques }\end{array}$ & $05 / 01 / 17$ & $\begin{array}{l}\text { Monde/ } \\
\text { France }\end{array}$ & 3 & $\begin{array}{l}\text { En Slovaquie, des miliciens } \\
\text { d'extrêmerdroite } \\
\text { patrouillaient dans les trains } \\
\text { pour chasser les roms et les } \\
\text { migrants. }\end{array}$ \\
\hline He garçon $4 \mathrm{C}$ & $\begin{array}{l}\text { Erasmus : } 30 \text { ans } \\
\text { d'échanges, } 5 \\
\text { millions r de } \\
\text { jeunes }\end{array}$ & $09 / 01 / 17$ & $\begin{array}{l}\text { Monde/ } \\
\text { Europe }\end{array}$ & 2 & $\begin{array}{l}\text { C'est le 30e anniversaire } \\
\text { d'Erasmus, une belle réussite } \\
\text { de la construction } \\
\text { européenne. }\end{array}$ \\
\hline
\end{tabular}




\begin{tabular}{|c|c|c|c|c|c|}
\hline $\begin{array}{l}\text { A. et Y. garçons } \\
4 \mathrm{~B}\end{array}$ & $\begin{array}{l}\text { Sefik, l'ancien } \\
\text { migrant, défend } \\
\text { un foot solidaire }\end{array}$ & $12 / 01 / 17$ & $\begin{array}{l}\text { Pays de la } \\
\text { Loire }\end{array}$ & 6 & $\begin{array}{l}\text { Un ex-réfugié bosniaque } \\
\text { organise un match de foot } \\
\text { avec des migrants. }\end{array}$ \\
\hline $\begin{array}{l}\text { B. garçon et } \mathrm{L} \text {. } \\
\text { fille } 4 \mathrm{C}\end{array}$ & $\begin{array}{l}\text { Toujours plus de } \\
\text { chrétiens } \\
\text { opprimés }\end{array}$ & $12 / 01 / 17$ & $\begin{array}{l}\text { Monde/ } \\
\text { France }\end{array}$ & 3 & $\begin{array}{l}\text { L'ONG protestante «Portes } \\
\text { Ouvertes» estime que } 215 \\
\text { millions de chrétiens sont } \\
\text { persécutés dans } 50 \text { pays, en } \\
\text { particulier en Asie. }\end{array}$ \\
\hline $\begin{array}{l}\text { Al et } \quad \mathrm{Ar} \\
\text { garçons } 4 \mathrm{C} \\
\text { (questionnaire } \\
\text { manquant) }\end{array}$ & $\begin{array}{l}4800 \text { migrants } \\
\text { reconduits en } \\
2016\end{array}$ & 20/01/17 & $\begin{array}{l}\text { Monde/ } \\
\text { France }\end{array}$ & 3 & $\begin{array}{l}4774 \text { personnes en situation } \\
\text { irrégulière ont été } \\
\text { renvoyées. La France abrite } \\
105000 \text { demandeurs d'asile. }\end{array}$ \\
\hline $\begin{array}{l}\text { N. et R. garçons } \\
\text { 4B }\end{array}$ & $\begin{array}{l}\text { Dix adolescents } \\
\text { jetés dans le } \\
\text { grand-froid }\end{array}$ & 23/01/17 & $\begin{array}{l}\text { Nantes } \\
\text { Métropole }\end{array}$ & \begin{tabular}{|l} 
Page \\
régionale
\end{tabular} & $\begin{array}{l}\text { La rigueur polaire n'a pas } \\
\text { assoupli les conditions } \\
\text { d'accueil des jeunes } \\
\text { migrants à Nantes, déboutés } \\
\text { de leur minorité si l'Aide } \\
\text { sociale à l'enfance du } \\
\text { département estime qu'ils } \\
\text { ont menti sur leur âge. }\end{array}$ \\
\hline $\begin{array}{l}\text { S. et T. garçons } \\
4 \mathrm{~B} \\
\text { S. et N. filles } 4 \mathrm{C}\end{array}$ & $\begin{array}{ll}\text {-Les } & \text { migrants de } \\
\text { retour à } & \text { Calais } \\
\text {-Plus } & \text { discrets, } \\
\text { les r migrants } \\
\text { reviennent à } \\
\text { Calais }\end{array}$ & 24/01/17 & France & $\begin{array}{l}\text { Une }+ \\
\text { suite p. } 5\end{array}$ & $\begin{array}{l}3 \text { mois après le } \\
\text { démantèlement de la } \\
\text { "jungle ", les migrants } \\
\text { tentent toujours de passer } \\
\text { en Angleterre, notamment } \\
\text { plusieurs centaines de } \\
\text { mineurs. }\end{array}$ \\
\hline 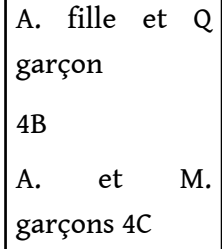 & $\begin{array}{l}\text { Immigration: } \\
\text { Trump barricade } \\
\text { l'Amérique }\end{array}$ & 26/01/17 & $\begin{array}{l}\text { Monde/ } \\
\text { Europe }\end{array}$ & $\begin{array}{l}\text { Une }+ \\
\text { suite p. } 2\end{array}$ & $\begin{array}{l}6 \text { jours après son élection, } \\
\text { Trump a décidé d'appliquer } \\
\text { ses mesures sécuritaires: } \\
\text { mur avec le Mexique et refus } \\
\text { d'accueillir les réfugiés. }\end{array}$ \\
\hline $\begin{array}{l}\text { L. garçon et C. } \\
\text { fille } 4 C\end{array}$ & $\begin{array}{l}\text { Les Chinois de } \\
\text { France en ont } \\
\text { assez du racisme }\end{array}$ & $27 / 01 / 17$ & $\begin{array}{l}\text { Monde/ } \\
\text { Europe }\end{array}$ & p.3 & $\begin{array}{l}\text { Entretien avec la présidente } \\
\text { de l'association chinois de } \\
\text { France à l'occasion du } \\
\text { nouvel an chinois }\end{array}$ \\
\hline E. et F. filles $4 \mathrm{C}$ & $\begin{array}{l}\text { Trump: } \\
\text { confusion dans } \\
\text { les aéroports }\end{array}$ & $30 / 01 / 17$ & $\begin{array}{l}\text { Monde/ } \\
\text { Europe }\end{array}$ & $\begin{array}{l}\text { Une }+ \\
\text { suite p. } 2\end{array}$ & $\begin{array}{l}\text { Pagaille et indignation dans } \\
\text { les aéroports du monde } \\
\text { entier suite à la décision de } \\
\text { Trump. }\end{array}$ \\
\hline
\end{tabular}




\begin{tabular}{|c|c|c|c|c|c|}
\hline $\begin{array}{l}\text { A. et LA filles } \\
4 \mathrm{~B}\end{array}$ & $\begin{array}{l}\text { Le décret Trump } \\
\text { affole les } \\
\text { européens }\end{array}$ & $31 / 01 / 17$ & $\begin{array}{l}\text { Monde/ } \\
\text { Europe }\end{array}$ & 2 & $\begin{array}{l}\text { Son texte anti-immigration } \\
\text { pourrait permettre de } \\
\text { consulter les données des } \\
\text { citoyens de l'UE. }\end{array}$ \\
\hline $\begin{array}{l}\text { M. et E. } \\
\text { garçons } \\
\text { 4B } \\
\text { G. et A. garçons } \\
\text { 4C }\end{array}$ & $\begin{array}{l}\text { Le décret Trump } \\
\text { angoisse les } \\
\text { réfugiés syriens }\end{array}$ & $07 / 02 / 17$ & $\begin{array}{l}\text { Monde/ } \\
\text { Europe }\end{array}$ & p.2 & $\begin{array}{l}\text { L'annulation du décret } \\
\text { Trump sur l'immigration a } \\
\text { permis à quelques familles } \\
\text { syriennes de rejoindre les } \\
\text { USA mais pour la majorité } \\
\text { des réfugiés syriens c'est } \\
\text { l'incertitude. }\end{array}$ \\
\hline $\begin{array}{l}\text { J. et E. filles } \\
4 B\end{array}$ & $\begin{array}{lr}\text {-Syrie : } & \text { des } \\
\text { milliers } & \text { de } \\
\text { détenus } & \\
\text { exécutés } \quad \text { (titre } \\
\text { en « Une ») } \\
\text {-Amnesty r } \\
\text { dénonce r la } \\
\text { «prison } & \\
\text { abattoir " } & \\
\text { d'Assad } \quad \text { (titre } \\
\text { p.2) }\end{array}$ & 08/02/17 & $\begin{array}{l}\text { Monde/ } \\
\text { Europe }\end{array}$ & $\begin{array}{l}\text { Une } \\
\text { suite } \\
\text { p.2 }\end{array}$ & $\begin{array}{l}20 \text { à } 50 \text { détenus assassinés } \\
\text { dans cette prison à Damas } \\
\text { depuis les } 4 \text { années de la } \\
\text { guerre civile en Syrie. Ces } \\
\text { opposants politiques sont } \\
\text { tous déclarés coupables lors } \\
\text { d'un semblant de procès. }\end{array}$ \\
\hline A. et L. filles $4 \mathrm{C}$ & $\begin{array}{lr}\text { Le } & \text { "collectif } ~ \\
\text { de } & \text { migrants } \\
\text { s'essaie } & \text { au } \\
\text { handball } & \end{array}$ & $16 / 02 / 17$ & $\begin{array}{l}\text { Pays de } \\
\text { Retz }\end{array}$ & $\begin{array}{l}\text { Page } \\
\text { régionale }\end{array}$ & $\begin{array}{l}\text { Grâce à une association } \\
\text { sportive de handball, des } \\
\text { jeunes demandeurs d'asile } \\
\text { pratiquant le football ont } \\
\text { essayé le handball. }\end{array}$ \\
\hline $\begin{array}{l}\text { Y.et N. garçons } \\
\text { 4B }\end{array}$ & $\begin{array}{l}\text { Afrique du Sud: } \\
\text { poussée } \\
\text { xénophobe }\end{array}$ & $27 / 02 / 17$ & $\begin{array}{l}\text { Monde/ } \\
\text { Europe }\end{array}$ & $\begin{array}{l}\text { Une } \quad+ \\
\text { suite p. } 2\end{array}$ & $\begin{array}{l}\text { L'économie va mal en Ads } \\
\text { les milliers d'étrangers } \\
\text { africains servent de boucs } \\
\text { émissaires à des marches } \\
\text { anti-immigrés. }\end{array}$ \\
\hline $\begin{array}{l}\text { L. et L. filles } \\
4 \mathrm{~B} \\
\text { F. et B. garçons } \\
4 \mathrm{C}\end{array}$ & $\begin{array}{l}\text { Migrants: la } \\
\text { terrible route } \\
\text { des Balkans }\end{array}$ & $01 / 03 / 17$ & $\begin{array}{l}\text { Monde/ } \\
\text { Europe }\end{array}$ & $\begin{array}{l}\text { Une }+ \\
\text { suite p. } 2\end{array}$ & $\begin{array}{l}\text { Depuis que la Grèce a fermé } \\
\text { ses portes, les migrants } \\
\text { tentent de passer par la } \\
\text { Bulgarie et le Serbie, avec } \\
\text { pour seule issue, l'entrée } \\
\text { illégale par la Hongrie. }\end{array}$ \\
\hline $\begin{array}{l}\text { M. et C.filles } \\
4 B \\
\text { B. et A. garçons } \\
4 C\end{array}$ & $\begin{array}{l}\text { Immigration : les } \\
\text { juges bloquent à } \\
\text { nouveau Trump }\end{array}$ & $17 / 03 / 17$ & $\begin{array}{l}\text { Monde/ } \\
\text { Europe }\end{array}$ & 2 & $\begin{array}{l}\text { La justice fédérale paralyse } \\
\text { un décret du président jugé } \\
\text { discriminatoire envers les } \\
\text { musulmans. }\end{array}$ \\
\hline
\end{tabular}




\begin{tabular}{|l|l|l|l|l|l|}
\hline $\begin{array}{l}\text { S garçon et T } \\
\text { fille 4B }\end{array}$ & $\begin{array}{l}\text { Aur, Royaume- } \\
\text { Uni,Européen } \\
\text { devientrun un } \\
\text { étranger }\end{array}$ & 20/03/17 & $\begin{array}{l}\text { Monde/ } \\
\text { Europe }\end{array}$ & $\begin{array}{l}\text { Une }+ \\
\text { suite p. 2 }\end{array}$ & $\begin{array}{l}\text { Depuis le référendum de juin } \\
2016 \text { où les Britanniques ont } \\
\text { décidé de quitter l'UE, les } \\
\text { Européens ne sentent plus } \\
\text { les bienvenus pour vivre et } \\
\text { travailler dans ce pays. }\end{array}$ \\
\hline D. et M. filles & $\begin{array}{l}\text { A St B, la vie } \\
\text { dans un centre } \\
\text { pour migrants }\end{array}$ & 20/03/17 & $\begin{array}{l}\text { Pays de } \\
\text { Retz }\end{array}$ & $\begin{array}{l}\text { Page } \\
\text { régionale }\end{array}$ & $\begin{array}{l}\text { En temps normal, le centre } \\
\text { accueille des vacanciers, des } \\
\text { salariés d'EDF et leur famille. } \\
\text { Depuis octobre, il héberge } \\
\text { des demandeurs d'asile. } \\
\text { Parmi eux, Nadir. }\end{array}$ \\
\hline
\end{tabular}

\section{Le déroulement}

19 La première séance visait à faire repérer par les élèves la structure d'un article de presse (titraille, chapô, illustration, légende, les $5 \mathrm{~W}$ : Who? Where? When? What? Why?). Un document réalisé par la documentaliste leur était distribué et servait d'appui à un travail sur un article du JDE (Journal Des Enfants) intitulé «Que deviennent les enfants de Syrie?». Le choix de cet article centré sur les enfants et les jeunes déplacés et réfugiés en zone de conflit semblait pouvoir permettre aux élèves, sinon de s'identifier, du moins de s'intéresser davantage au thème des migrations internationales.

20 A l'issue de ce travail collectif de repérage, les élèves étaient invités à parcourir par groupes de deux, un des exemplaires de Ouest-France pré-sélectionnés par les auteurs de cet article, afin d'y trouver un article traitant des migrants et de l'analyser quant à sa forme et son contenu.

21 Le jour de la deuxième séance, les élèves ont choisi eux-mêmes leur article en feuilletant le quotidien qui leur avait été distribué. Ce temps de feuilletage nous a permis de leur faire conscience qu'un quotidien régional contenait également des rubriques internationales et nationales et que l'on pouvait trouver des articles sur les migrants dans toutes. Lorsque des articles étaient repris par la « Une », ils constataient que ces articles faisaient partie de l'actualité la plus «brûlante ». Cette « vitrine » du journal est définie par le " rédacteur en chef ».

22 Il nous semblait important qu'ils fassent eux-mêmes ce travail de sélection et de repérage en se confrontant au journal, à ses rubriques, etc. Le problème est qu'ils n'ont pas forcément sélectionné les articles qu'ils n'associaient pas "naturellement " aux «migrants »: ce fût le cas par exemple des articles «Erasmus, 30 ans d'échanges, 5 millions de jeunes » ou " Toujours plus de chrétiens opprimés... » Ils ont surtout choisi ce qui «faisait écho» pour eux dans la presse du moment: les mesures antiimmigration de Donald Trump. Cependant, nous les avons parfois orientés vers des articles qu'ils n'auraient pas sélectionnés eux-mêmes: "Afrique du Sud: poussée xénophobe » par exemple ou les deux articles cités précédemment. 

élèves devaient coller la photocopie de leur article (et de sa une éventuelle) sur le
panneau adéquat et justifier son choix parmi les quatre accrochés aux murs : « qui sont
les migrants?»; «le 'voyage' des migrants »; «les principales raisons des migrations
internationales »; " "l'accueil des migrants : hostilité ou solidarité? ?. Nous avions
choisi ce plan de classement des articles lors de l'élaboration du corpus. Chaque groupe
d'élèves devait ensuite analyser à l'oral son article en fonction des « $5 W »$.

Documents distribués aux élèves (CDI collège Frida Kahlo - Christel Maridet)

\section{1-La structure d'un article de presse}

Un article de presse, qu'il soit tiré d'un quotidien d'information, d'un magazine, ou d'un site Internet, est toujours structuré de la même façon. Il comporte :

UN TITRE : donne une information, il doit être court et attirer l'attention. Il peut être :

Informatif, il résume de façon objective le contenu de l'article (ex : « Deux fillettes victimes de la tempête »)

Accrocheur, frappant, il attire l'attention du lecteur, il cherche à surprendre ou amuser (ex : « Le Salon de la pêche en mer veut appâter le public »)

Partisan, il laisse apparaître l'opinion de celui qui l'écrit (ex : « Accident de voiture à cause d'un automobiliste fort imprudent. »)

UN CORPS DE TEXTE : plus ou moins long, il doit comporter :

Une accroche : introduction d'1 ou de 2 phrases destinées à retenir l'attention du lecteur.

Les $5 \mathrm{~W}$ : Who, What, when, Where, why et éventuellement How.

Cette règle de base permet de donner rapidement et avec clarté l'essentiel d'une information.

Des intertitres : permettent d'aérer l'article quand celui-ci est trop long.

Ce sont quelques mots en gras qui peuvent découper l'article en paragraphes, permettant au lecteur de construire son parcours de lecture.

Une chute : conclusion courte qui termine l'article en l'élargissant ou en ouvrant sur un autre sujet.

À noter : l'article peut avoir plusieurs formes : la brève, le compte-rendu, le reportage, l'interview, l'enquête, l'éditorial, le billet d'humeur... 
Il est toujours écrit selon un angle, c'est la façon dont on va traiter le sujet.

UN SURTITRE et/ou UN SOUS-TITRE : se trouvent soit au-dessus, soit en dessous du titre. Ils apportent des informations complémentaires au titre :

Surtitre : où? Quand?

Sous-titre : comment ? Pourquoi ? (ex : Disparus d'Orvault : un mystère orchestré ?)

Titre + surtitre + sous-titre $=$ La titraille

UN CHAPEAU (ou CHAPO) : texte court en haut de l'article, en gras, qui résume l'essentiel de l'information et qui doit accrocher l'attention du lecteur.

On dit qu'il « coiffe » l'article. Il peut répondre aux $5 \mathrm{~W}$

Who (Qui sont les personnes dont on parle dans l'article?)

What (Quelle est l'information principale ? Que s'est-il passé ?, de Quoi parle l'article?)

When (Quand s'est déroulée l'information? A quelle date fait référence l'article ?)

Where (Où se passe l'information, Quels lieux sont mentionnés dans l'article ?)

Why (Pourquoi ? Quelles sont les causes de l'information?) ou How (Quelles sont les conséquences de l'information? Comment l'expliquer ?)

UNE PHOTO ou un DESSIN : c'est une illustration qui complète l'article et qui ne se contente pas de l'illustrer. Elle informe et explique, elle peut choquer ou émouvoir.

Elle est toujours accompagnée d'une légende.

\section{Le questionnaire}

\section{Revue de presse thématique : « Les migrants »}

Une revue de presse thématique fait la synthèse des articles de presse concernant un thème. Elle permet d'avoir une vision globale d'un sujet; ici, les migrants et nous permettra de répondre aux questions suivantes :

- Qui sont-ils ? De quels pays viennent-ils?

- Quelles sont les principales raisons des migrations internationales?

- Pourquoi ont-ils décidé de quitter leur pays natal?

- Quel a été leur « voyage » ? Ont-ils dû faire appel à des « passeurs » ?

- Comment ont-ils été accueillis ? Hostilité ou solidarité? 


\section{Présentation du périodique :}

Journal ou magazine?

NOM du journal?

Périodicité ? (Quotidien ? Hebdomadaire ? Mensuel ?...)

Date :

Place de l'article dans le périodique :

L'article est-il représenté à la Une du journal ? Si oui qu'en déduisez-vous?

Dans quelle rubrique se trouve-t-il ?

À quelle page?

Le titre de l'article : Écrivez le titre. Est-il informatif, accrocheur ou partisan? Justifiez votre réponse

Sous-titre : Y en a-t-il un ? Si oui écrivez-le.

La photographie : Que voit-on sur la photographie ? Décrivez-là !

La légende : Écrivez le texte de la légende. Est-elle utile pour comprendre l'image? Pourquoi ?

Le chapô : Écrivez le texte du chapô de votre article

LE CORPS DE L'ARTICLE :

Retrouvez les $5 \mathrm{~W}$ en vous aidant aussi du chapô

Who?

What?

When?

Where? (Ville/pays/continent)

Why?

À quelle question votre article répond-il ? Justifiez en quelques lignes

Après validation, allez coller votre article sur le panneau qui correspond à votre article et préparez-vous à le présenter à l'oral pour la prochaine séance presse.

\section{Problèmes rencontrés et améliorations possibles}

Les élèves étaient peu familiers de la lecture de la presse, en particulier de la presse papier. Se sont posés à eux des problèmes pratiques que nous n'aurions pas 
soupçonnés, par exemple : où commence l'article ? Certains ont confondu le début de la lecture de l'article avec la légende de l'image. Qui le signe ? La lecture et le feuilletage n'allaient pas soi pour tous. Davantage de séances de feuilletage et d'analyse de la presse seraient bienvenues et pas seulement au cycle 4 , en faisant réaliser une revue de presse régulièrement par exemple dès la $5 \mathrm{e}$.

Mais les élèves avaient aussi des problèmes d'ordre cognitif pour comprendre réellement le contenu des articles. Peu habitués à lire l'actualité, ils maîtrisaient souvent mal le vocabulaire utilisé par les journalistes : «bouc émissaire " par exemple, se trompaient dans la géographie des pays cités (Érythrée situé en Europe...), inversaient parfois l'ordre des causes et des conséquences («L'économie va mal car les gens disent que c'est à cause des immigrés »). Cette mauvaise utilisation de la conjonction de coordination montre aussi leurs propres préjugés concernant la réalité des migrants. Ces préjugés entendus à la maison, nous les avons retrouvés dans plusieurs questionnaires mais aussi lors de l'évaluation finale. Par exemple dans un questionnaire, tel élève explique que «Trump veut barricader l'Amérique pour éviter que les migrants s'incrustent ». Tel autre élève était surpris que son article aborde la question de la xénophobie entre Africains. Pour lui, ces discriminations ne pouvaient être que le fait de Blancs envers les Noirs ou le contraire.

Pour autant, les classes du collège sont très hétérogènes et d'autres élèves ont bien perçu et analysé la distinction entre un titre informatif, accrocheur ou partisan. Par exemple, ceux travaillant sur le titre "Plus discrets, les migrants reviennent à Calais » ont écrit que ce titre était partisan car la discrétion "peut être une qualité ou un défaut. Il donne son jugement sur l'apparition des migrants. » A l'inverse, un groupe travaillant sur « Trump sème la confusion dans tous les aéroports » a trouvé ce titre à la fois informatif, accrocheur et partisan, en justifiant chacune de ces trois dimensions.

Dans leurs réponses au questionnaire d'analyse, les élèves n'ont pas bien compris la consigne concernant le classement de leur article dans une problématique plus générale: Qui sont les migrants? Quelles sont les raisons des migrations internationales? Quel a été leur voyage? Comment ont-ils été accueillis? Hostilité ou solidarité ? Ils ont crû que nous leur demandions d'inventer une question à laquelle répondrait leur article, ce qui était d'ailleurs intéressant. Mais la consigne devra être plus précise une prochaine fois. Cependant, certains élèves ont bien compris cette question et, mieux, qu'on attendait d'eux qu'ils réévaluent leurs présupposés sur les migrations. Ainsi, l'article "Toujours plus de chrétiens opprimés dans le monde" pouvait être rangé dans la rubrique «les principales raisons des migrations internationales». Mais un groupe a fait un choix plus pertinent, en expliquant que «notre article répond à la question: "Qui sont les migrants". Parce que les migrants peuvent être chrétiens. »

29 À propos du « trajet » des migrants, nous envisageons de leur faire tracer sur une carte le chemin parcouru, du pays d'origine au pays mentionné par l'article. Ce document à compléter en plus du questionnaire permettrait d'éviter les contre-sens géographiques.

Les analyses de leurs articles ont donc été assez superficielles dans l'ensemble, ce qui s'est aussi ressenti lors de leur prestation orale. Ils se contentaient souvent de reprendre les « $5 \mathrm{~W}$ » et d'y répondre de manière très scolaire. Nous n'avons pas eu l'impression que les groupes s'étaient énormément investis. La réalité des migrants est restée au rang de "question » abstraite, comme si ces articles ne décrivaient pas une réalité concrète. Les questions «What» et "Why» ont été souvent survolées: par 
exemple à la question censée expliquer pourquoi un foyer de vacances local accueille des demandeurs d'asile, les élèves répondent : " parce qu'ils ne trouvent pas de foyer ». À la décharge des élèves, le temps imparti pour l'analyse des articles au CDI était souvent trop court. Nous aurions dû faire une troisième séance avant l'oral et corriger leurs questionnaires afin de rectifier les contre-sens, les imprécisions, etc.

31 L'évaluation finale, quant à elle, est intervenue les 11 et 12 mai, soit sept semaines après le début du travail sur la presse et les migrants. Entre temps, un cours sur les migrations avait eu lieu en classe durant 5 à 6 heures. Cela signifie donc que les définitions de réfugié, de migrant ou autre n'étaient pas connues de la plupart des élèves au moment des séances sur la presse. Plus généralement, il leur manquait les connaissances de base sur la géographie des migrations (chiffres, principaux flux, etc.). Cela peut expliquer les difficultés cognitives déjà évoquées dans l'analyse de l'article de presse et le poids des préjugés. Mais comment expliquer que ces préjugés demeurent lors de l'évaluation finale? En effet, si les résultats ont été satisfaisants en termes de connaissance des définitions ou des localisations, ils l'ont été nettement moins pour l'identification, la compréhension fine et la prise de distance critique vis-à-vis du document proposé (un article de presse). Plus surprenant, une petite moitié d'élèves a répondu à une question de cours en donnant leur avis personnel, comme lors du débat organisé en EMC, alors que la réponse attendue avait été écrite dans les cahiers en classe. Cette question était: «quelles sont les conséquences des migrations dans les pays d'arrivée (en Europe)?» Elle figurait sur la fiche de révision distribuée une semaine auparavant.

Une dizaine d'élèves de chacune des classes a fait les réponses suivantes, reproduites ici avec leurs fautes :

« Faire perdre de l'argent au commerce situer où les migrants arrivent. / Il peuvent provoquer des atenta / manque de travaille ; atentat ; pauvreté / Plus d'habitation donc habitations beaucoup plus serrées; plus de pollution; Ca crée encore plus de chômage / Moins de travail / Baiser le chiffre d'affaire; Moins en moins de touriste; Leur mise en valeur par rapport au autre / Beaucoup moins de travail pour les Européens ; moins de touristes / Ils peuvent faire fuir les voyageur / Les gens veulent être gentil avec eux donc ils font baisser les prix (pas de grosse récolte d'argent) / Il y a trop de monde dans les pays; Les magasins perdent beaucoup d'argents / Les restoran perde 10 à $15 \%$ des bénéfise / Les voiajer n'y ron plus dans les pays ou il y a des migrans / peuvent faire perdre de la clientelle / Surpopulation, trop d'immigrés dans un pays et pas assez dans un autre / Ils y plus de personne au chomage / Les migrants prennent les emplois d'autres personnes; Ils sont trop personnes à loger / Sa peut provoquer que certaines personne quitte leur pays car il en on marre des migrants; Provoquer la guerre / Pour les commerce perdre de l'argent. » Extraits de copies d'élèves de $4 \mathrm{e}$.

Le signes [;] sépare les réponses d'un même élève. Le signe [/] sépare les réponses de deux élèves.

Sans doute aurait-il fallu partir de ces préjugés des élèves pour ouvrir la séquence, afin de les confronter ensuite aux réalités dont rend compte la presse. La seule occasion pour eux d'exprimer leur sensibilité et leur opinion sur les migrations fût le débat organisé en EMC le mercredi 3 mai, soit en fin de séquence. Mais leur échec à cette question tient aussi à leur difficulté, déjà mentionnée, à différencier les causes des conséquences. 


\section{Conclusion}

Nous avons souvent eu le sentiment lors de ce travail, qui nous a demandé beaucoup de préparation et particulièrement à la collègue documentaliste, que "la montagne accouchait d'une souris ». Non seulement les élèves n'étaient pas familiers de la presse, mais «l'actualité des migrants» ne les touche pas directement dans leur environnement rural. D'un autre côté, nous nous sommes dit que nous avions eu d'autant plus raison de travailler cette actualité. Nous envisageons d'ailleurs d'aborder le thème des migrants en $4 \mathrm{e}$ l'an prochain avec "collège au cinéma ", dont deux des trois films mettent en scène des migrants, et en $5 \mathrm{e}$ avec un spectacle de théatre. Varier les approches sur cette réalité permettra peut-être à nos élèves "d'ouvrir les yeux " avec plus de bienveillance sur le monde qui les entoure.

Les objectifs n'ont donc pas tous été atteints, mais au moins un motif de satisfaction demeure. Presque tous les élèves ont été capables de comprendre la structure d'un article de presse et de transposer cette connaissance dans un travail réalisé parallèlement en histoire. Il s'agissait de se mettre à la place d'un journaliste envoyé en Algérie au début du XXe siècle et de rédiger un article de presse sur l'organisation de cette société coloniale. Cette tâche complexe proposée par le manuel Nathan (2016) a été bien mieux réalisée grâce au travail fait lors de la semaine de la presse. Cette remarque finale renvoie elle aussi à la nécessité de varier les approches et les supports.

\section{NOTES}

1. Source : www.education.gouv.fr.

2. Circulaire $n^{\circ}$ 2017-051- Bulletin officiel de l'éducation nationale (BOEN) $n^{\circ} 13$ du 30 mars 2017.

3. Toutes les citations du paragraphe suivant proviennent de l'arrêté du 9 novembre 2015, publié dans le BOEN spécial nº 11 du 26 novembre 2015.

4. Toutes les citations de ce paragraphe proviennent de l'arrêté du 12 juin 2015, publié dans le BOEN spécial $n^{\circ} 6$ du 25 juin 2015.

5. Cf. note 3 .

6. Cf. note 3 .

\section{INDEX}

Index géographique : France

Mots-clés : enseignement secondaire, méthodologie 


\section{AUTEURS}

CHRISTEL MARIDET-TJON-A-TAI

professeure / documentaliste

Christel.Maridet@ac-nantes.fr

ANTOINE DUMONT

professeur d'histoire-géographie

Antoine.Dumont@ac-nantes.fr 